\section{PIEDAD DE LA CIERVA: UNA SORPRENDENTE TRAYECTORIA PROFESIONAL DURANTE LA SEGUNDA REPÚBLICA Y EL FRANQUISMO}

\author{
Inmaculada Alva Rodríguez \\ Universidad de Navarra \\ ialva@unav.es
}

Cómo citar este artículo/Citation: Alva Rodríguez, I. (2016). Piedad de la Cierva: una sorprendente trayectoria profesional durante la segunda república y el franquismo. Arbor, 192 (779): a322. doi: http://dx.doi.org/10.3989/arbor.2016.779n3012

Recibido: 29 septiembre 2014. Aceptado: 26 noviembre 2014.

RESUMEN: Piedad de la Cierva obtuvo la licenciatura de Ciencias por la Universidad de Valencia en 1932. Ese mismo año se trasladó a Madrid para realizar su tesis doctoral en el prestigioso Instituto Rockefeller. Dos años más tarde le fue concedida una beca de ampliación de estudios en el Niels Böehr de Copenhague para profundizar en materias relacionadas con la radioactividad. Después de la guerra civil pudo continuar con su investigación científica trabajando en temas de los que fue pionera como el vidrio óptico o la fabricación de ladrillos refractarios. El objetivo de este trabajo es dar visibilidad al trabajo desarrollado por esta pionera reconstruyendo su biografía intelectual a través de lo que ella recoge en sus memorias y los escasos datos que nos proporcionan la bibliografía relacionada. A través de su labor científica quedarán de manifiesto las dificultades que encontró y los apoyos que le facilitaron desenvolverse en un mundo entonces preferentemente masculino

PALABRAS CLAVE: Piedad de la Cierva; ciencia; género; Junta para la Ampliación de Estudios; Consejo Superior de Investigaciones Cientificas.

\section{PIEDAD DE LA CIERVA: A SURPRISING CAREER DURING THE SECOND REPUBLIC AND FRANCO'S REGIME}

Copyright: (C) 2016 CSIC. Este es un artículo de acceso abierto distribuido bajo los términos de la licencia Creative Commons Attribution (CC BY) España 3.0.

ABSTRACT: Piedad de la Cierva earned her degree in Sciences from the University of Valencia in 1932. That same year she moved to Madrid to prepare her thesis at the prestigious Rockefeller Institute. Two years later, she was given a grant to extend her studies on radioactivity at the Nils Bohr Institute in Copenhagen. She was able to continue her scientific research after the Civil War and was a pioneer in areas such as optical glass or refractory brick manufacture. This work aims to raise the profile of this female pioneer's work by tracing her intellectual biography from her memoirs and the limited data available from the specialised bibliography. Her work as a scientist revealed the difficulties that she met and the support she obtained to help her make her way in a predominantly male world.

KEYWORDS: Piedad de la Cierva; sciences; gender; Junta para la Ampliación de Estudios; Consejo Superior de Investigaciones Científicas. 
Piedad de la Cierva dejó por escrito a la edad de ochenta años sus recuerdos personales, centrándose sobre todo en su carrera profesional ${ }^{1}$. "He llegado hasta aquí suavemente, casi sin darme cuenta y dejo atrás un largo pasado, que voy a recordar un poco duro a veces; pero lleno de sentido, de trabajo, de alegría y de paz" (Cierva Viudes, 1993, fol. 1), diría en el comienzo de esas memorias. Era consciente de que había sido testigo y protagonista de unos años excepcionales en los que las mujeres estaban accediendo a la educación universitaria y empezaban a desarrollar su carrera profesional. Estos escritos autobiográficos de Piedad constituyen un material de gran valor para conocer de primera mano no solo su recorrido profesional, sino también sus personales sentimientos y vivencias. Precisamente, este tipo de memorias o "life writings" es a menudo la única fuente para reconstruir la historia de las mujeres, tan invisibles habitualmente en las historias y registros oficiales (cf. Lerner, 2006, p. 13). Trataré por tanto de presentar la trayectoria de Piedad de la Cierva fundamentalmente al hilo de sus recuerdos.

Piedad de la Cierva obtuvo la licenciatura de Ciencias por la Universidad de Valencia en 1932. No era normal entonces que las mujeres alcanzaran los estudios universitarios, aunque ya se había dado un avance importante en los de bachillerato. En el curso que Piedad de la Cierva acabó la carrera, el número de mujeres matriculadas en la universidad española era de 2.206, es decir, representaban un $6 \%$ del total de alumnos universitarios (cf. Montero, 2009, p. 205). Fue una de esas mujeres afortunadas porque su padre se dio cuenta, tras la Gran Guerra, de que el mundo había cambiado y las mujeres estaban destinadas a protagonizar un mayor papel (cf. Cierva Viudes, 1993, fols. 9-10). Pensó que la mejor manera de capacitar a su hija era facilitándole una educación universitaria. Juan de la Cierva hubiera preferido que estudiara Farmacia, quizá porque en aquel entonces se veía como una carrera adecuada para las mujeres (cf. Capel Martínez, 1986, pp. 461, 472; Flecha García, 2015, p. 64), pero su hija Piedad manifestó desde el principio una clara inclinación por la Química. Finalmente, su padre accedió a que se matriculara en la Facultad de Ciencias de la Universidad de Murcia a condición de que hiciera también las asignaturas que le faltaban para obtener Magisterio². Piedad reflejaba en sus memorias que fue su profesor de Ciencias quien despertó en ella el amor por la Química. Mostraba con su elección -en contra del parecer de su padre y de las ideas predominantes en su tiempo- una clara determinación y personalidad puesto que optaba por una carrera en la que estaría en minoría y en la que las perspectivas profesionales para una mujer eran escasas. Carmen Magallón ha puesto de relieve que para estas mujeres pioneras en el campo de las ciencias experimentales el apoyo paterno fue determinante, aunque debían tener también "dos rasgos de carácter, necesarios en aquellas circunstancias: una disposición clara a ejercer su libertad, por encima de convencionalismos, y el no cejar ante las dificultades" (Magallón Portolés, 2010b, p. 323). Rasgos que se daban en Piedad quien, por ejemplo, se negó a asistir a clase con una señora de compañía, como su padre pretendía conforme a los usos de la época (cf. Cierva Viudes, 1993, fol. 6).

El 2 de octubre de 1928 empezó a estudiar como alumna oficial en la Universidad de Murcia. Era un mundo nuevo para ella, acostumbrada a estudiar en casa con profesores particulares, refugiada en el cariño de su madre que paliaba la severidad paterna, exigiéndole constantemente la excelencia académica. "Las exigencias de mi padre -recuerda en sus memorias- a pesar de su buena intención, me habían proporcionado años muy duros que prefiero no recordar. También sus planes económicos familiares, en lo que mi madre no podía intervenir, creaban un ambiente poco agradable. Metida en casa, poco trato con gente de mi edad, con un plan de vida diferente, yo era tímida y encogida. En esos años, mi amparo era mi madre, que también sufría mucho" (Cierva Viudes, 1993, fol. 12). Fue su madre la que le transmitió una vida cristiana de piedad sencilla que mantendría toda su vida.

En la Universidad de Murcia era la única mujer, una situación que se repetiría más veces en su vida. En escasas ocasiones sufrió discriminación por ser mujer, posiblemente porque la avalaban sus aptitudes intelectuales, además de que, en general, se encontró con profesores que le ayudaron en su promoción profesional. Así, por ejemplo, siempre recordaría gratamente el trato recibido en las clases de Física del profesor Fernando Ferrando, un hombre a quien volvería a encontrar de nuevo en la Universidad de Valencia (cf. Cierva Viudes, 1993, fol. 13).

El siguiente curso, 1929-30, se trasladó a Valencia para continuar la carrera. Era también una oportunidad de salir del asfixiante ambiente familiar y vivir nuevas experiencias. En primer lugar, pudo encontrar otras chicas universitarias en la Residencia de Escolapias en la que se alojaba, lo que rompía en parte el aislamiento que había sufrido en Murcia. El internado de las monjas escolapias era para chicas de bachillerato, pero admitían también a universitarias. Junto con Piedad de la Cierva había tres estudiantes de Magisterio y dos de Medicina. 
El mundo femenino de la residencia ofrecía un agudo contraste con la facultad de Químicas donde nuevamente era la única mujer de su clase, aunque había cinco chicas más en la Facultad. La vida en la universidad y en las calles de la ciudad era agitada y convulsa con algaradas callejeras y huelgas. Tras la proclamación de la segunda república y el peligro de que la residencia de escolapias fuera asaltada, las monjas se deshicieron del internado. Lo que en principio parecía una contrariedad para Piedad de la Cierva se convirtió en una nueva oportunidad de conocer un ambiente más selecto.

El profesor Ferrando, que se había trasladado a Valencia en 1931, le ofreció alojamiento con su familia. Allí se encontró con un ambiente cristiano similar al de su casa, pero presidido por una mayor libertad. La mujer de Ferrando era además Licenciada en Filosofía y Letras y archivera: "El ambiente de aquella casa era totalmente distinto al de mi familia y al que todas las familias que yo conocía. Se trataban de igual a igual, con una gran cordialidad y delicadeza. Comentaban lo que decían los periódicos; yo nunca había leído ninguno. Comentaban la quema de conventos, lo que hacía el gobierno, etc." (Cierva Viudes, 1993, fol.18).

Cuando acabó la carrera en junio de 1932 con Premio Extraordinario de Licenciatura tenía diecinueve años y ningún deseo de dar por concluida su carrera científica. Como le ocurriría en otras ocasiones, encontró el aliento adecuado en sus profesores. Fue el profesor de Química General, Antonio Ipiens ${ }^{3}$, quien le propuso hacer el doctorado en Madrid. Para ello le dio una carta de recomendación dirigida a Julio Palacios, uno de los investigadores más prestigiosos de la Universidad Central de Madrid.

Si ya Valencia había significado una apertura de horizontes, la marcha a la capital de España era una nueva aventura preñada de grandes perspectivas. El Madrid de los años treinta destacaba por ser una ciudad de gran movimiento cultural, político, intelectual, lo que se ha llamado la Edad de Plata. Muchos de los protagonistas de esos años han sido objeto de estudio y son bien conocidos en la comunidad científica, literaria y artística, pero no ocurre así con las mujeres. Aunque sus nombres aparecen a veces en antologías, estudios científicos, etc., no han recibido todavía una atención adecuada. Poetas y escritoras como Ernestina de Champourcín, Rosa Chacel o Carmen Conde, artistas como Maruja Mallo o filósofas como María Zambrano tienen escasas monografías y no se les integra correctamente dentro del contexto cultural e intelectual en el que vivieron ${ }^{4}$. Lo mismo ocurre con las mujeres científicas. En las diversas instituciones de la Junta de Ampliación de Estudios (JAE) había un grupo de mujeres que aportaron importantes descubrimientos y fueron pioneras en diversos campos científicos. Pero también es cierto que "de estas pioneras españolas en las ciencias experimentales hay estudios contextuales, prosopográficos, de significado generacional y grupal, en los que se dan a conocer sus nombres y contribuciones, pero falta la profundización en su experiencia individual, el conocer más sobre sus trayectorias y circunstancias vitales, en relación con su incorporación a la ciencia" (Magallón Portolés, 2010a, p. 767) $)^{5}$. Piedad de la Cierva, ciertamente fue una de estas pioneras. Este ensayo es una aproximación a su obra científica y una primera semblanza intelectual a partir sobre todo de lo recogido en sus recuerdos personales, con lo que se quiere contribuir a llenar ese vacío al que hacía referencia Magallón Portolés.

\section{LOS INICIOS: LA SECCIÓN DE RAYOS X EN EL INSTITUTO ROCKEFELLER}

"A lo largo de mi vida he ido recorriendo etapas que, cada vez, me llevaban a ambientes más elevados social y culturalmente" (Cierva Viudes, 1993, fol. 21). Así iniciaba en sus memorias su llegada a Madrid en octubre de 1932. Como alojamiento eligió la Residencia de la Institución Teresiana, un proyecto innovador de promoción de la mujer, fundado por el sacerdote Pedro Poveda (cf. Montero, 2009, pp. 77-95; 213-217). Allí convivió con otras treinta y cinco universitarias.

Pero fue su llegada al recién estrenado Instituto Rockefeller lo que le causó más impacto 6 . "La llegada con mi padre al Instituto Rockefeller me deslumbró [...]. Era el Instituto un gran edificio modernísimo, con instalaciones estupendas para investigar en distintos ramos de Física y Química. Había sido promovido por la Junta de Ampliación de Estudios e instalado con la más completa equipación después de visitar varios grandes Centros de Investigación de Europa" (Cierva Viudes, 1993, fol. 21). Avalada por la carta de presentación de Ipiens, Julio Palacios se hizo cargo de ella. Le organizó el plan de estudios que consistiría en asignaturas de doctorado en la Universidad y trabajo en el Instituto para aprender las Técnicas de Rayos $\mathrm{X}$, como preparación de su futura tesis doctoral (cf. AGUCM, Expediente académico de Piedad de la Cierva, 59/99$41,3)^{7}$. Además debería repasar francés e inglés para poder utilizar la bibliografía.

El equipo de Julio Palacios en la Sección de Rayos $X$ estaba formado por dos físicos: Rafael Salvia y Luis Bru (que preparaban oposiciones); Julio Garrido, doc- 
tor en Ciencias Naturales, José María González Barredo, químico, Jorge Doetsch, Ingeniero de Minas, José Losada y Piedad de la Cierva. Nuevamente era la única mujer y en este caso, además, la persona más joven. Palacios era uno de los pioneros en los estudios de cristalografía en España. Precisamente en 1926 se había publicado el primer trabajo de investigación relacionado con la cristalografía de Rayos X en la revista Anales de la Real Sociedad Española de Física y Química, "Determinación de la estructura cristalina del óxido de níquel, del cobalto y sulfuro de plomo", firmado por una mujer, Felisa Martín Bravo, -que fue la primera doctoranda en Ciencias Físicas- (cf. Magallón Portolés, 2001, pp. 46-49), dirigida por Julio Palacios. La creación de la Sección de Rayos $X$ en el Rockefeller en 1932 bajo la dirección de este y el equipo ya mencionado dio el impulso definitivo a los estudios cristalográficos mediante la difracción de rayos $\mathrm{X}$ (cf. Martínez Ripoll, 2009, p. 228).

Rafael Salvia fue el encargado de enseñar a los recién llegados -Losada y De la Cierva-. Les introdujo en el estudio de estructuras cristalinas por difracción de Rayos $\mathrm{X}$, les enseñó a manejar los aparatos de medición, a revelar películas, a medir diagramas y a hacer cálculos. "Recuerdo mi gran sorpresa cuando comprobé que yo era capaz de calcular la distancia entre los átomos de cloro y sodio de un cristal de sal. Y cómo me impresionaba pensar que Dios, Creador del Universo, había distribuido los átomos, tan pequeños de aquella forma tan asombrosa" (Cierva Viudes, 1993, fol. 24), recordaba Piedad al cabo de los años.

Conforme se fue familiarizando con el edificio empezó a conocer a otras mujeres que trabajaban en las demás secciones del Instituto. Piedad de la Cierva recordaba expresamente a Amelia Garrido y a Narcisa Martín Retortillo con las que les uniría una amistad que duró toda su vida. Desde 1932 hasta que se clausuró la actividad del edificio a causa de la guerra, en 1937, fueron 36 las científicas vinculadas al centro. Entre ellas se podría destacar -además de Piedad de la Cierva- a Carmen González Escobar, Dorotea Barnés, Pilar Madariaga, Rosa Bernís o María Teresa Salazar y otras muchas $^{8}$. Aunque la desproporción en número era clara, representaban el $25 \%$ de los científicos que trabajaban en el Rockefeller, tanto los hombres como las mujeres contribuyeron al crecimiento en cantidad y calidad del trabajo científico (cf. González Ibáñez y Santamaría García, 2009, pp. 383-386). Tenían además por costumbre reunirse en el salón de té donde se celebraban reuniones una vez por semana para conocerse y tratar temas de interés (cf. Magallón Portolés, 2007, pp. 221-227).
La actividad de Piedad de la Cierva durante estos años de formación e investigación fue muy destacable. Una vez acabadas las asignaturas de doctorado, se le nombró ayudante de clases prácticas de Química Orgánica de la Facultad de Ciencias. Empezó a participar en congresos internacionales, como el IX Congreso Internacional de Química Pura y Aplicada que reunió a sabios del mundo entero, celebrado en la primavera de 1934. La comunicación que presentó exponía algunos de los resultados que serían luego la base de su tesis doctoral.

Ese mismo año, ya en noviembre, se centró en el tema de su tesis doctoral, "Factores químicos del azufre y del plomo". Recordaría siempre con agradecimiento la dedicación y paciencia del profesor Julio Palacios en la elaboración de su tesis quien, entre bromas y veras, le decía "Piedad, cuando acabe la tesis, tendrá que leerse el Quijote" (Cierva Viudes, 1993, fol. 28). Pues sus dotes como investigadora no tenían correlación con su capacidad como escritora. El resto del curso trabajó en colaboración con Luis Rivoir en un método de análisis de acero con Rayos X. Se publicó en Anales de las Sociedades Españolas de Física y Química. Durante unos meses trabajó con ellos una física argentina, Cecilia Mossín -considerada la primera física argentina-. Fruto del trabajo de esos meses publicó con José Losada "Factor atómico del zinc". Esas relaciones de trabajo se convirtieron de nuevo en una relación de amistad como lo prueba el hecho de que se vieran en París, donde continuaba trabajando Cecilia, en mayo de 1935 en la parada que hizo Piedad en la ciudad, camino de Copenhague.

Piedad de la Cierva fue una las mujeres -entre las investigadoras de esta sección- que realizó una aportación más relevante y continuada durante este periodo (cf. Magallón Portolés, 2004, pp. 240-241). Sus trabajos darían lugar a la tesis doctoral Los factores atómicos del azufre y del plomo y a siete artículos que se publicarían en los Anales de la Sociedad Española de Física y Química, algunos de ellos en colaboración con Julio Palacios o con otros investigadores que trabajaban en la Sección de Rayos $X^{9}$.

A principios de 1935, había ya defendido su tesis con la que obtuvo la calificación de sobresaliente y Premio Extraordinario de doctorado (cf. AGUCM 59/99-41, 3). Julio Palacios le propuso que solicitara una beca para ampliar estudios en el extranjero. En principio solicitó una a la Junta de Ampliación de Estudios para estudiar con el profesor Marck en Viena (cf. Residencia de Estudiantes. Archivo de la JAE, http://archivojae.edadeplata.org/jae_app/JAE/36-514). Quizá no se la dieron 
porque finalmente obtuvo una beca de la Academia de las Ciencias de la Fundación del Marqués de Cartagena para trabajar en temas de radioactividad en el Niels Böehr, Instituto de Física teórica de Copenhague junto al profesor George von Hevesy, descubridor del hafnio y futuro Premio Nobel (cf. AGA, (5) 19.1 32/13636, leg. $9619 / 3)^{10}$.

\section{LA EXPERIENCIA DANESA: LA RADIACIÓN ARTIFICIAL}

El Instituto de Física Teórica, fundado por Niels Böehr era uno de los centros más prestigiosos en materia de radioactividad artificial. Böehr había recibido el Premio Nobel en 1913, con 28 años, por descubrir la estructura del átomo. Era un lugar privilegiado al que acudían investigadores de toda Europa y se trabajaba con otros centros de Alemania, Inglaterra y Francia. El prestigio científico de quienes trabajaban allí era muy alto y algunos eran ya o serían Premios Nobel. Muchos llegaban huyendo de la Alemania nazi, buscando un lugar en el que poder trabajar con libertad. Entre ellos, el húngaro George von Hevesy, Otto Frisch, de Viena o el alemán James Frank. Trabajaban unas treinta personas, todos hombres excepto una judía alemana, la doctora Selvy, con la que Piedad de la Cierva trabajó estrechamente. La materia principal de trabajo era la desintegración artificial del átomo que había descubierto el matrimonio Juliot-Curie.

El ambiente del Colegio de la Asunción donde se alojaba era muy selecto. Además de las alumnas, vivían tres rusas, parientes del zar que estaban allí asiladas. Las monjas eran veinte y de nacionalidades muy distintas. Nada más llegar, le entregaron un Catecismo en francés y la llave de la residencia para que tuviera libertad de entrar y salir. Cada alumna tenía una tutora y Piedad rememoraba con cariño y admiración las conversaciones que mantenía con la suya, la Madre Hildegarda. Una mujer muy culta, que había vivido muchos años en Filipinas y aficionada a la física: "se ofreció a darme clases de alemán y charlábamos de vez en cuando, comentando las maravillas de Dios que había inventado y creado los átomos" (Cierva Viudes, 1993, fol. 36).

El Niels Böehr le resultó muy austero en comparación con el Instituto Rockefeller. Pero allí se estaba trabajando en los fundamentos de lo que sería la energía nuclear. Aprendió métodos revolucionarios y el manejo de instrumentos que posibilitaban la desintegración artificial del átomo. Sus estudios se centraron en el aluminio y el bromo. Aplicaba las técnicas que le enseñaban y discutía los resultados con Hevesy, Frisch y Bronsted. Se asombraba de la excelencia intelectual del sitio donde trabajaba, que era también el ambiente que presidía la ciudad: "Dinamarca era un país de alto nivel social, prácticamente una clase única. Todos los niños, incluidos los príncipes, asistían a la escuela pública. Solamente había una especie de aristocracia: la del estudio" (Cierva Viudes, 1993, fol. 41).

La estancia de Piedad de la Cierva en Copenhague fue muy fructífera y tendría como resultado la publicación de dos artículos en los Anales de las Sociedades Españolas de Física y Química con el título: "Bifurcación en la transmutación del aluminio por la acción de neutrones rápidos" y "Separación de los hisopos del Bromo". Además aprovechó las vacaciones de Navidad para visitar el Instituto de Radio en París. El 30 de enero de 1935, Irene Joliot le enseñaba las instalaciones del centro y tuvieron oportunidad de hablar y debatir sobre sus trabajos comunes (cf. Cierva Viudes, 1993, fol. 43).

También el viaje de vuelta a España, cuando acabó el período de la beca, fue organizado por el profesor Hevesy para que pudiera conocer otros institutos de Química con los que le convendría mantener el contacto. Uno de ellos fue el laboratorio de la Dra. Lise Meitner, pionera de la fisión nuclear, en el Kaiser Wilhelm Institut fur Chemie de Berlín ${ }^{11}$.

Su vuelta a España estaba llena de nuevos proyectos e ilusiones. Empezó a montar un contador de partículas $\beta$ y se puso un equipo a su disposición para poner en práctica todo lo aprendido en materia de radioactividad artificial, sumándose así el Rockefeller a los centros pioneros en estos temas. Con este objeto había recibido otra beca de la Academia de Ciencias para continuar en Madrid esos trabajos (cf. AGA, (5) 19.1 32/13636, leg. 9619/3). Pero corría el año 1936 y el 18 de julio terminó con todos esos sueños.

\section{EL INSTITUTO DE ÓPTICA “DAZA DE VALDÉS”}

Cuando el conflicto bélico acabó, Piedad de la Cierva intentó incorporarse a su actividad ordinaria en el Rockefeller. Durante la guerra había trabajado como profesora de física y química en un instituto de Osuna y tuvo que esperar a acabar el curso académico para trasladarse definitivamente a la capital de España. Esta nueva etapa científica de Piedad, aunque mencionada en algunos libros, es menos conocida que su época de la JAE (cf. Alcalá Cortijo y Magallón Portolés, 2008, pp. 155, 161-162). Es por eso que la principal fuente de investigación por el momento son las memorias inéditas que he estado utilizando hasta ahora. 
En 1939 se fundó el Consejo Superior de Investigaciones Científicas (CSIC) que integraba la mayor parte de los centros de la JAE. En principio, el panorama no parecía muy favorecedor para la integración de las mujeres en este instituto científico. Su primer presidente, Julio Casares, se oponía a la admisión de mujeres. Además se nutrió casi exclusivamente de catedráticos, "estamento casi exclusivo de varones que no facilitaba en muchos casos que las mujeres hicieran estudios de doctorado" (Alcalá Cortijo, 1996, p. 63). Sin embargo, la presencia de mujeres en el CSIC fue más significativa de lo que podía parecer. De hecho, como señala Alcalá Cortijo- el $10 \%$ de las mujeres que figuraban en la plantilla del CSIC en los años setenta habían entrado en la década de los cuarenta. Eran en general, mujeres que se habían formado en los años treinta, no habían sufrido la depuración o el exilio y eran necesarias en una institución que estaba en momento de crecimiento (cf. Alcalá Cortijo, 1996, pp. 63-64).

Este fue el caso de Piedad de la Cierva. Cuando llegó a Madrid, los distintos centros se estaban reorganizando y la sección Rayos $X$ aún no funcionaba (cf. González Ibáñez y Santamaría García, 2009, p. 36). Fue entonces cuando contactó con ella José María Otero Navascués, a quien había conocido en los meses que ambos habían estado refugiados en la embajada de Noruega (cf. Pérez Fernández-Turégano, 2012, pp. 37-38). Le propuso participar en el proyecto de creación de una sección de Óptica dentro del Instituto de Física para impulsar la fabricación de instrumentos ópticos para la Marina. Pronto se formó un grupo con dos ingenieros industriales: Antonio Santamaría y Alberto Navascués; un físico, Armando Durán, además de Otero y Piedad de la Cierva (cf. Pérez FernándezTurégano, 2012, pp. 75-76). Sería el núcleo del futuro Instituto de Óptica "Daza de Valdés", que Navascués dirigiría desde 1946 hasta 1966 (cf. Pérez FernándezTurégano, 2012, p. 54).

Es claro que el prestigio y los conocimientos de Piedad de la Cierva sobre la radiación artificial eran demasiado importantes para ignorarla, ayudado por el hecho de que no se hubiera significado políticamente a favor de la República en los años treinta y que su familia mantuviera lazos políticos y de amistad con los nuevos dirigentes. Fue la única mujer admitida en la primera constitución del CSIC. Aunque pronto irían llegando otras, como María Teresa Vigón, María Egüés y Olga María Riquelme porque ni Otero Navascués, ni Julio Palacios o Armando Durán, tenían inconveniente en trabajar con mujeres (cf. Moya de Guerra et al., 2002, pp. 561-562). Miraban a las personas por su valía profesional.
Sin embargo, fue necesario tomar precauciones. Así, cuando el profesor Durán propuso a María Egüés, en marzo de 1940, que participara en un cursillo de Óptica Geométrica y Cálculo de Sistemas que se impartiría en la sección de Óptica a cargo del profesor Weidert, le advirtió que al acabar el curso debía marcharse del edificio porque Julio Casares no quería mujeres en su instituto. Más de una vez tuvo que esconderse debajo de la mesa porque se acercaba Casares (cf. Moya de Guerra et al., 2003, p. 4). María Egüés recordaba agradecida el apoyo de sus compañeras y, sobre todo, el de Piedad de la Cierva que también hacía el curso. Una de las veces que Julio Casares se la encontró le advirtió que no quería verla por allí fuera de las horas del cursillo. Le confesó que tenía que tolerar la presencia de Piedad de la Cierva porque llevaba mucho tiempo trabajando allí y conocía a su familia, pero que con María no tenía esos compromisos. Otero y Durán buscaron una solución intermedia para que Egüés pudiera seguir trabajando, como ya habían tenido que hacer con Piedad. Todo cambió cuando Albareda se hizo cargo del CSIC y las mujeres pudieron entrar con libertad a trabajar allí en los distintos institutos (cf. Moya de Guerra et al., 2002, p. 564).

En cualquier caso, la presencia de estas mujeres en el CSIC y en un campo, el de las ciencias químicas, en el que eran una minoría, evidencia cierta continuidad respecto a la época de la segunda república. Cuestiona en cierto modo la idea universalmente admitida de que el franquismo supuso una ruptura con respecto a los logros conseguidos para la mujer en la época republicana o al menos hace pensar que esa ruptura no fue tan radical como se venía pensando.

Además de sus tareas en el Instituto de Óptica, Piedad de la Cierva fue nombrada Auxiliar de cátedra de la asignatura "Estructura atómico-molecular y Espectroscopia" que se impartía en los cursos de Doctorado. Consuelo Flecha ha puesto de relieve que fue este el medio habitual por el que las mujeres empezaron a dar clases en la universidad, ya desde la segunda república. Como ayudantes de clases prácticas o auxiliares de cátedra, en ocasiones mal pagadas, se perpetuarían en esos puestos sin muchas esperanzas de promoción. Incluso -como fue el caso de Piedad- se ocuparon de las clases teóricas en casos de cátedra vacante. De hecho, ninguna de las que empezaron antes de 1936 llegaron a tener su plaza en propiedad y apenas quedan registros de su actividad en los documentos oficiales (cf. Flecha García, 2010, p. 260).

La cátedra en la que empezó a ayudar Piedad de la Cierva había sido creada para Miguel Catalán. Piedad 
pensaba que le serviría lo aprendido en Copenhague y además aprendería mucho de Catalán pues asistiría a sus clases, al tiempo que le ayudaba en las prácticas. Sin embargo, Catalán había sido depurado y se le impidió acceder a su cátedra. Para su sorpresa, se encontró con que era ella la que tenía que ocuparse de las clases teóricas, pero sin que constara oficialmente en ningún sitio y sin recibir un sueldo. Había cursado las asignaturas y la base de sus estudios en Copenhague le resultaban muy útiles, pero preparar las clases le suponía mucho esfuerzo. Catalán la asesoró, y todos confiaban en que se tratara de una situación transitoria pero no fue así ${ }^{12}$. Los alumnos eran unos treinta. Entre ellos había licenciados de antes de 1936, sacerdotes, catedráticos de instituto, auxiliares de universidad. Hasta entonces, Piedad de la Cierva no había sufrido ninguna dificultad seria por el hecho de ser mujer en el mundo científico en que se movía. Posiblemente, su apellido le facilitó también el camino, pero fue sobre todo su solvencia científica e intelectual lo que le permitió moverse con soltura en un mundo preferentemente masculino.

El fracaso en las oposiciones a cátedra a las que se presentó en 1941, como se verá en el apartado siguiente, le provocó un enorme desencanto pues le hizo darse cuenta de que el mundo universitario era aún un coto cerrado en el que las mujeres se encontraban en situación de inferioridad. De hecho, estaba ocupando una cátedra vacante por la que percibía una cantidad mínima, el tercio del sueldo anual de lo que ganaba un catedrático y sin reconocimiento oficial pues constaba solo como auxiliar de cátedra (cf. AGUCM, P. 746, 44 y P. 469, 12).

Decidió, por tanto, dejar las clases prácticas de la Complutense y centrarse en el Instituto de Óptica donde trabajaba a gusto y en temas que le interesaban. Allí se ocupaba de preparar espejos de aluminio por evaporación al vacío, una técnica necesaria para la fabricación de prismáticos (cf. Cierva Viudes, 1993, fol. 71).

En el comienzo del curso 1941-42 fue nombrada Directora del Laboratorio de la antigua Residencia de Señoritas de las calle Fortuny. Pero sobre todo, se dedicó al Instituto de Óptica "Daza de Valdés" donde permanecería hasta 1950. De los setenta y ocho científicos que trabajaron en el Instituto durante los diez años que duró, veinte eran mujeres, entre las que destacaban Olga Mạ Riquelme, Teresa Vigón o Narcisa Martín Retortillo. Pero realmente las que se mantuvieron trabajando allí todos esos años fueron solo Piedad de la Cierva y María Egüés (cf. González Ibáñez y
Santamaría García, 2009, pp. 392-393). El número de publicaciones de esos años da fe del intenso trabajo que Piedad desarrolló durante esos años ${ }^{13}$.

\section{LAS OPOSICIONES A CÁTEDRA DE UNIVERSIDAD}

Una de las pocas veces que sufrió las consecuencias de la discriminación de género fue en unas oposiciones a cátedra de universidad. A principios de 1941 se habían convocado las oposiciones de cátedra de Físico-Química para las universidades de Sevilla, Valencia y Murcia (cf. AGA, (5) 19.1 32/13636, leg. 9619/3). Piedad de la Cierva se presentó ante la insistencia de su padre que soñaba con que su hija fuera la primera mujer catedrática de Universidad (cf. Cierva Viudes, 1993, fol. 67).

Además de Piedad, se presentaba otra mujer, María Teresa Salazar Bermúdez. Nacida en Villanueva del Ariscal (Sevilla), era ya Doctora en Químicas en 1931. De 1931 a 1934 trabajó en la Sección de Físico-Química del Rockefeller y en 1934 obtuvo una beca de la JAE para estudiar la "Estructura del núcleo atómico" en Francia. Siguió trabajando luego en el Instituto Nacional de Física y Química y fue una de las más cercanas colaboradoras del Dr. Moles. Antes de la Guerra Civil ya había publicado cinco artículos científicos (cf. Magallón Portolés, 1996, p. 59 y Montero, 2009, p. 266).

Las dos tenían méritos suficientes para presentarse a la convocatoria con garantías de éxito. Otero Carvajal, que ha estudiado las oposiciones a cátedras universitarias durante el franquismo, asegura a la vista de los expedientes, que sus curricula eran incluso superiores a los de algunos de los candidatos (cf. Otero Carvajal, 2012, p. 59). Además de Piedad de la Cierva y Teresa Salazar se presentaban Octavio Rafael Foz Gazulla, Julián Rodríguez Velasco y José María González Barredo.

Antes de presentarse a los exámenes, para los que Piedad se había estado preparando duramente, se enteró por un amigo de su padre y por el ministro de educación, Ibáñez Martín, que los resultados ya estaban dados. Además existía una especie de acuerdo entre las facultades de no dar la plaza a una mujer. Ibáñez Martín le aconsejó que no se presentara. Era mejor hacerlo para cátedra para instituto donde con toda seguridad se le daría una plaza (cf. Cierva Viudes, 1993, p. 68). A pesar de todo, las dos mujeres se presentaron.

Las actas de las sesiones del tribunal reflejan las diferencias de trato que sufrieron Piedad de la Cierva y Teresa Salazar. En la calificación de uno de los ejer- 
cicios, por ejemplo, se comenta lo mismo de las dos: "acordando por unanimidad el que a pesar de las deficiencias observadas por el Tribunal por la Srta. opositora podía continuar con el siguiente ejercicio". También la valoración de sus trabajos científicos fue negativa. Así, dos de los vocales coincidían en minusvalorar las publicaciones de Piedad de la Cierva porque habían sido hechas en colaboración con otros, sin considerar el prestigio de los colaboradores como Julio Palacios o Hevesy, quien ganaría el Premio Nobel en 1943. Solo el profesor Ipiens, que conocía personalmente a Piedad y apreciaba su labor científica, opinaba que "la autoridad del profesor Palacios es garantía del valor de las citadas publicaciones, en las que la Srta. de la Cierva revela una enorme aptitud para las investigaciones físico-químicas. Sus restantes trabajos sobre radioactividad artificial, algunos bajo la dirección del profesor Hevesy constituyen una iniciación en las delicadas técnicas de la desintegración artificial de los átomos" (AGA, (5) 19.1 32/13636, leg. 9619/3).

Las cátedras de Sevilla y Valencia las ganaron dos de los opositores masculinos y la de Murcia se declaró desierta. Parecía que preferían dejarlo así antes que dársela a una mujer. Teresa Salazar presentó una instancia pidiendo la revisión de las calificaciones. Argumentaba que el Tribunal no era apto para examinarles porque ninguno era profesor de físico-química. Pero además manifestaba su sospecha de que no se le hubiera dado la plaza por ser mujer, cuando -según decía- algunos de los que habían estado entre el público habían calificado sus exposiciones de brillantes. Se quejaba finalmente del daño hecho a su prestigio por el hecho de haber dejado una plaza desierta. El recurso fue desestimado argumentando que se presentaba fuera de plazo (AGA, (5) 19.1 32/13636, leg. 9619/3).

No era la primera vez que Teresa Salazar había sufrido esta negativa experiencia. Se había presentado ya en 1936 a las oposiciones de la Universidad de La Laguna y lo volvería a intentar, de nuevo sin éxito, en 1945 para las de Valencia y Valladolid (cf. Flecha García, 2010, p. 272). Piedad de la Cierva, por el contrario, desistió de volver a intentarlo más, decepcionada por la discriminación sufrida. La realidad es que no sería hasta 1953 cuando una mujer ganó las oposiciones a cátedra. María de los Ángeles Galino sería la primera mujer catedrática, en Historia de la Pedagogía e Historia de la Pedagogía española, por la Universidad de Madrid ${ }^{14}$.

\section{LA CREACIÓN DEL LTIEMA Y LA AVENTURA DEL VIDRIO ÓPTICO}

En 1945 se creó el LTIEMA (Laboratorio y Taller de Investigación del Estado Mayor de la Armada), cuyos fondos se dedicaron sobre todo a la investigación del vidrio óptico. Josemaría Otero Navascués fue nombrado subdirector del centro y director en 1948 (cf. Pérez Fernández-Turégano, 2012, p. 52). En cuanto se hizo cargo del Instituto impuso su impronta personal. Buscó, por ejemplo, la colaboración con otros centros nacionales e internacionales. Quería además que el LTIEMA fuera "un laboratorio de investigación y desarrollo, cubriendo actividades desde la ciencia aplicada hasta el desarrollo del prototipo" y un pequeño taller industrial. La línea prioritaria sería la óptica, electrónica y comunicaciones, dirección de tiro, mecánica de precisión y metrología (cf. Pérez Fernández-Turégano, 2012, pp. 52s).

Para este nuevo proyecto quiso contar con Piedad de la Cierva, quien sería contratada ese año como Personal Técnico Civil de la Marina. Además de las tareas de investigación que desempeñó, se encargaba de las sesiones prácticas de química a los alumnos de la Escuela de Ingenieros Navales. La relación con los alumnos era muy cordial e incluso se fraguaron amistades que se mantuvieron en el tiempo como Manuel Bernardo y Adolfo García Abriles, quienes Ilegaron a ser Almirantes (cf. Cierva Viudes, 1993, fol. 74).

Pero su tarea principal era el trabajo sobre las láminas antirreflectoras que recubrían la superficie de las lentes y los prismas, permitiendo así la visión nocturna. Otero estaba interesado en la fabricación en España de estos instrumentos ópticos desde su vuelta de Alemania donde había probado su eficacia. Para Piedad suponía un nuevo reto, puesto que era un trabajo pionero en España y había muy poca bibliografía. Los avances fueron a base de muchos ensayos y experimentaciones.

Finalmente a partir de 1945 pudo aplicarse ese procedimiento a los prismáticos que se fabricaban en el LTIEMA. Piedad de la Cierva presentó la Memoria de los trabajos realizados a la Academia de las Ciencias en 1946. Esa Memoria recibió el Primer Premio de la Academia de Ciencias para trabajos de investigación. Era la primera mujer que recibía un reconocimiento de este grado (cf. Cierva Viudes, fol. 76). Los primeros resultados sobre las láminas reflectoras se dieron a conocer en un congreso en París. En 1947 acudió también a la Feria de Muestras de Barcelona para exponer los prototipos fabricados en el Instituto: sextantes, diferentes tipos de prismáticos, telémetros. Colaboraban con ella María Teresa Vigón, Xula Vigón y María Egüés. 
Otro de los grandes proyectos de Otero fue la industrialización en España del vidrio óptico, un material que se producía aún en pocos lugares del mundo. Quería que el LTIEMA fuera un centro pionero en esta materia. Encomendó esta tarea a Piedad de la Cierva para lo que la envió a Estados Unidos en 1948 con el fin de que estudiara de primera mano los secretos de esa industria. Piedad trabajó en el National Bureau of Standards en Washington y visitó la Facultad de Ingeniería de Vidrio de la Universidad de Toledo (Ohio) y otras fábricas de vidrio óptico, como la Libby Owens, especializada en la fabricación del vidrio irrompible, por entonces desconocido en España. Por último, antes de volver a España estuvo en la Bausch and Jomb Optical y la Kodak en Rochester (Nueva York) (cf. Cierva Viudes, 1993, fols. 81-98).

De vuelta a España y con la experiencia adquirida, se puso a trabajar en la fabricación del vidrio, tipificando primero las materias primas que debía utilizar, construyendo los hornos y formando un equipo compuesto por investigadores y obreros. Una de las investigadoras era Francisca de Andrés - que ya había trabajado en otras ocasiones con Piedad de la Cierva-. Los primeros estudios sobre las materias primas, en concreto las arcillas, sirvió a De Andrés como tema de tesis doctoral ${ }^{15}$. Aunque la directora real era Piedad de la Cierva, tuvo que pedirle a José María Albareda que figurara como director, ya que Piedad no era catedrática (cf. Cierva Viudes, 1993, fol. 103).

Finalmente consiguió fabricar el primer ensayo de vidrio óptico en 1954. La Memoria se presentó a un Concurso del Consejo Superior de Investigaciones Científicas, con el título "Ensayos de fabricación de vidrio óptico". Fue premiada con el 10 Premio de Investigación técnica "Juan de la Cierva" en 1955 y publicada por el Consejo Superior de Investigaciones Científicas $^{16}$.

Acudió en 1956 al Congreso Internacional celebrado en Madrid sobre "Realidad en estado sólido", junto con otros tres compañeros -Francisca de Andrés, Aurea Perales y José Rodríguez- donde presentaron dos trabajos: "Influencia de electrolitos en el colado de crisoles" y "Atacabilidad de los crisoles por el vidrio fundido", que luego fueron publicados.

La fama y el prestigio de Piedad de la Cierva y sus colaboradores trascendían las fronteras: recibían peticiones de separatas de sus artículos desde la fábrica de Bausch and Lomb y también del Instituto del Vidrio de París. En junio de 1956 visitó las instalaciones del LTIEMA el Dr. Heindl de Wash, quien elogió sus tra- bajos sobre arcillas y vidrios y le pidió que publicase los resultados de sus ensayos en revistas de Estados Unidos. Piedad empezó también a colaborar en el Instituto del Vidrio de La Granja ayudando en la selección de materias primas (cf. Cierva Viudes, 1995, fol. 10).

Otero Navascués había dejado la dirección del LTIEMA en 1956. Los nuevos directores no estaban interesados en la fabricación del vidrio óptico y en 1957 se decidió desmontar los hornos para añadir esos terrenos al campo de deporte.

Compatible con los trabajos rutinarios en el LTIEMA, como, por ejemplo, los análisis de aceros, Piedad de la Cierva empezó a interesarse por otros temas de investigación -al tener que abandonar el tema del vidrio óptico- con los que además pudo ayudar e iniciar en la investigación a otras mujeres: las superficies pulidas del vidrio y la capacidad aislante de la cascarilla de arroz.

En el otoño de 1959 había acudido a ella Luisa Arroyo, del Instituto de Electrónica, para pedirle que le dirigiera la tesis doctoral. En principio, Luisa Arroyo quería trabajar sobre los vidrios conductores transparentes, pero Piedad le sugirió que lo hiciera sobre las superficies pulidas por estar más relacionado con el trabajo que estaba haciendo. Juntas realizaron un trabajo muy original que sirvió a Luisa Arroyo de tesis doctoral: "Superficies pulidas de vidrio". Parte de esos resultados fueron en un artículo conjunto ${ }^{17}$. Como ya había hecho antes, tuvo que figurar como Director de la tesis doctoral un catedrático universitario.

A través de una revista americana había conocido las investigaciones sobre unos ladrillos aislantes, fabricados con cenizas de paja de arroz, que despertó su curiosidad. Ese artículo le había traído algunos recuerdos de la infancia, en concreto, las barras de hielo que se compraban en su casa y eran transportadas cubiertas en paja de arroz. Empezó a experimentar por su cuenta con la cascarilla del arroz. No se quemaba de forma normal sino en los hornos de vidrio, que por entonces aun no se habían desmontado. Se quemaba la parte orgánica pero quedaban unas cenizas muy blancas y abundantes que conservaban la misma estructura de la cascarilla. Las analizó al microscopio, hizo fotografías e inició un análisis químico de densidad aparente y real. Buscó bibliografía sobre el tema y se dio cuenta de que tenía un nuevo tema de investigación. "El examen microscópico de las cenizas de cascarilla daba una imagen preciosa: una cedillas huecas de sílice casi pura proporcionaban un material ideal como refractario y como aislante. A 
mí me asombraban mis observaciones y me divertía considerar una vez más de las maravillas de la creación: en medio del campo cubierto de agua, donde se cultiva al arroz, aquellas ramitas endebles chupan del suelo la sílice, que se va colocando ordenadamente, mezclada con la parte vegetal, que primero es verde y luego leñosa. Así se va formando la cascarilla alrededor de cada granito de arroz, blanco y arenoso" (Cierva Viudes, 1995, fols. 10-11).

Ante la falta de interés por parte del Ministerio de Marina por seguir con la industrialización del vidrio óptico, Piedad de la Cierva se implicó cada vez más en este nuevo proyecto de investigación. Veía que "era un tema de estudio interesante porque podía tener aplicación inmediata para la fabricación de ladrillos refractarios aislantes, para las calderas de los barcos y para otros hornos como los de cemento que en aquella época de gran impulso de la construcción se importaban de Dinamarca" (Cierva Viudes, 1995, fol. 12). El nuevo director del LTIEMA se interesó en esta nueva investigación y animó a Piedad de la Cierva a seguir. Le dio autorización para que colaboraran con ella dos mujeres que trabajaban en el laboratorio del LTIEMA con cierta regularidad: Antonia Muñoz y Guadalupe Ortiz de Landázuri.

El trabajo desarrollado sirvió de tesis doctoral a Guadalupe Ortiz de Landázuri, que fue leída en 1965 y obtuvo la calificación de sobresaliente cum laude. El trabajo se presentó a la convocatoria de los premios de Investigación "Juan de la Cierva" como trabajo en equipo en la opción "Investigación Técnica”. Obtuvieron el Premio y ya desde el LTIEMA se procedió a conseguir la patente del proceso de fabricación (cf. Cierva Viudes, 1995, fol. 20; Eguíbar, 2001, pp. 216-219). En este caso el "director" de la tesis fue el profesor Ángel Viau, muy amigo de Piedad de la Cierva.

Un nuevo director del LTIEMA imprimió una nueva orientación al instituto, dejando de lado la investigación. El trabajo de Piedad de la Cierva era cada vez más rutinario y con menos espacios para desarrollar líneas de investigación. Prácticamente se dedicaba a redactar normas sobre tipos de materiales que se debían utilizar en la Marina. La situación en el trabajo, unido al fallecimiento de uno de sus hermanos en 1969 y el agravamiento de la enfermedad de su madre, le llevaron a plantearse la jubilación anticipada, a los 63 años. Después de 35 años de trabajo en el LTIEMA iniciaba su jubilación el 1 de marzo de 1976 una mujer que había sido pionera en varios campos de las ciencias químicas.
Sus últimos años fueron una lucha contra la pérdida de memoria. Los recuerdos escritos por ella, que me han permitido reconstruir gran parte de su vida, dan fe de su confianza en el poder de la ciencia pero también el reconocimiento de sus propias limitaciones: "Muchas veces me he preguntado cómo se producen en nosotros las ideas, los pensamientos. Ya sé que el Espíritu Santo dirige nuestros pensamientos y acciones y Él me encomiendo con frecuencia. Pero no he visto nunca la relación entre el alma humana -el entendimiento, la voluntad, la memoria- y el funcionamiento del cuerpo, concretamente del cerebro. No sé si es problema de Psicología, de Fisiología... Desde hace algún tiempo noto que me falla la memoria: se me olvidan los nombres de personas muy conocidas; se me olvida lo que tengo que hacer o donde he guardado un papel. El médico dice que es falta de riego cerebral; un proceso de envejecimiento normal...Dicen que los ordenadores, ese invento nuevo que ya sobrepasa mi capacidad de aprender, funcionan de un modo parecido a la memoria... No sé cómo funcionan los ordenadores. Los científicos de hoy los han inventado y los fabrican. Y yo sigo con la curiosidad de saber cómo funcionan esas neuronas, de las que no conozco más que el nombre, y que son capaces de producir y almacenar vivencias de ochenta años" (Cierva Viudes, 1993, fol. 49).

\section{CONCLUSIONES}

El trabajo científico de Piedad de la Cierva no ha tenido aún el reconocimiento que merece. Este breve ensayo quiere ser un inicio de lo que será un proyecto más amplio, escribir la biografía de esta pionera científica. Experta en radiación artificial cuando estaba en sus inicios, su trabajo en la industrialización del vidrio óptico y el descubrimiento de las capacidades de la cascarilla de arroz para fabricar material refractario son algunos de sus logros que se vertieron en un número importante de artículos, algunas patentes y premios de investigación.

Es llamativo que hasta el momento no haya habido estudios sobre la labor desarrollada por esta científica, lo que es una muestra más de la persistente invisibilidad de la mujer a nivel académico. Las escasas menciones sobre su colaboración con Julio Palacios, Miguel Catalán y sobre todo con José María Otero Navascués suponen un agudo contraste con las monografías dedicadas a estos hombres de ciencia. Es, por tanto, una tarea pendiente rescatar del olvido el trabajo de Piedad de la Cierva y tantas otras mujeres que desarrollaron una labor destacada entre la segunda república y el franquismo. 
La actividad científica que desarrolló en dos períodos tan distintos manifiesta la existencia de una continuidad en su trabajo. No se puede hablar, por tanto, de una ruptura total entre ambas épocas. Junto a Piedad, encontramos otras mujeres que habían empezado su trayectoria profesional en los años anteriores a la guerra y que pudieron continuarla durante el franquismo. Arroja cierta luz sobre la situación de la mujer en este período y permite entrever que, a pesar de que se diera una tendencia a promover el trabajo de la mujer en el hogar, hubo mujeres que pudieron desarrollar sin cortapisas una labor científica importante.

En este sentido, también habría que destacar las redes de apoyo y colaboración mutua que se dieron entre estas mujeres en ambos períodos. En el caso de Piedad, hay que valorar su impulso y ayuda a otras investigadoras que estaban empezando, como prue-

\section{NOTAS}

[1] Estas memorias se encuentran en el Archivo General de la Prelatura del Opus Dei, Series U.1.4. Citaré este manuscrito como Cierva Viudes. Está dividido en dos documentos. El primero, con fecha 24 de octubre de 1993, consta de 103 folios escritos a mano por una sola cara. El segundo, fechado en 20 de marzo de 1995, son 24 folios mecanografiados también por una cara. En esas memorias describió también su encuentro con el Opus Dei. Fue una de las primeras mujeres que solicitó la admisión en el Opus Dei como Agregada en diciembre de 1952. Desde ese año hizo compatible su dedicación al Opus Dei con su trabajo como científica.

[2] Las alumnas que completaban el bachillerato podían obtener el título de Magisterio en un curso si se matriculaban de unas asignaturas, en concreto, Pedagogía, Música y Labores. Un buen estudio de la evolución de los estudios de Magisterio de 1900 a 1930 puede verse en Capel Martínez, 1986, pp. 424428.

[3] El profesor Antonio Ipiens Lacasa era catedrático de Química General en la Universidad de Valencia desde 1924. En 1941 obtuvo la cátedra de Química experimental en la Universidad de Madrid. Cf. http://www.ranf.com/acad\%C3\%A9micos/ acad\%C3\%A9micos-de-n\%C3\%BAmero/anteriores/1187-excmo-sr-d-antonio-ipi\%C3\%A9ns-lacasa.html. Su mujer también tenía una carrera universitaria, lo que explica que viera normal que Piedad de la Cierva continuara desarrollando una carrera científica, y la apoyara.

[4] Un interesante estudio sobre las intelectuales de la Segunda República que pone de relieve el desconocimiento de la importante actividad que desarrollaron es García Jaramillo (2013). Quizá el estudio más clásico y completo hasta el momento sea el de Mangini (2001).

[5] Hay algunos estudios detallados de estas científicas pioneras que permiten al menos hacerse una idea de la cantidad y ban las tres tesis doctorales que dirigió a tres mujeres, aunque no pudiera quedar constancia de ser la directora. Sería interesante estudiar hasta qué punto este tipo de redes se extendieron entre las mujeres investigadoras, aunque sean difíciles de rastrear, porque habitualmente, al no ser catedráticos de universidad, no pudieron firmar los trabajos que dirigieron.

La trayectoria científica de Piedad de la Cierva es de gran interés no solo por el valor en sí mismo de sus aportaciones a la ciencia sino porque plantea interrogantes sobre la situación de la mujer entre los años treinta y cuarenta y cuestiona la idea extendida de las escasas oportunidades que podía tener una mujer que quisiera desarrollar una carrera profesional. Invita por tanto a profundizar en el trabajo de esta pionera científica y de otras mujeres que, como ella, supieron abrirse paso con brillantez en un mundo preferentemente masculino.

calidad de las mujeres que a través de las becas de la JAE y otro tipo de pensiones contribuyeron al avance de la ciencia en distintos ámbitos. En este sentido es un clásico el estudio de Magallón Portolés (2004), en especial las páginas dedicadas al Instituto Nacional de Física y Química (pp. 223-260), donde realiza una interesante prosopografía de las 36 mujeres que trabajaron allí entre 1931-1936.

[6] El Instituto Rockefeller se inauguró el 6 de febrero de 1932. Llamado así por su principal benefactor, era el Instituto Nacional de Física y Química, dependiente de la Junta de Ampliación de Estudios. Un completo estudio de su trayectoria y logros científicos están recogidos en González Ibáñez y Santamaría García (2009).

[7] Ver el apartado Fuentes al final del artículo.

[8] Una breve biografía de algunas de estas mujeres puede leerse en Magallón Portolés, 1996, pp. 56-59. Un estudio más reciente es el de Merino Hernández, 2015, pp. 225-241.

[9] Esos artículos eran Cierva Viudes y Losada (1933), Cierva Viudes y Palacios (1934), Cierva Viudes y Palacios (1935), Cierva Viudes (1936), Palacios, Rivoir y Cierva Viudes (1936), Cierva Viudes y Rivoi (1936).

[10] Ver el apartado Fuentes al final del artículo.

[11] Una breve pero interesante biografía sobre esta física, que tan relacionada estaría posteriormente con la construcción de la bomba atómica, es Lewin Simen (1997).

[12] Miguel Catalán fue un destacado científico que con sus descubrimientos ayudó a científicos como Böehr a definir la estructura esencial del átomo. Era jefe de la Sección de Espectroscopia en el Instituto Rockefeller. Simultaneaba esos trabajos con la enseñanza como catedrático en la Universidad Complutense. 
Tras la Guerra Civil sufrió la depuración y no pudo recuperar su cátedra hasta 1946. Tampoco pudo reiniciar su actividad científica hasta 1950. Un completo estudio de su vida y obra es el de Sánchez Ron (1994). Es curioso que no se mencione a Piedad de la Cierva en la etapa en que sustituyó a Catalán en su cátedra, dado que el propio científico, asumiendo las circunstancias políticas, la estuvo asesorando.

[13] Los artículos publicados fueron Cierva y Durán (1941), Cierva (1943), Cierva y Durán (1943), Cierva (1944), Cierva y Cacho (1949).

[14] En la Revista Española de Pedagogía, donde se dio la noticia, se afirmaba: "ha sido eliminado el prejuicio, mantenido por algunos, de la inconveniencia de que una cátedra universitaria sea

\section{FUENTES}

Los archivos utilizados para esta investigación han sido el Archivo General de la Prelatura del Opus Dei (AGP) donde se encuentran las memorias de Piedad de la Cierva, base fundamental de este trabajo. En el Archivo General de la Administración (AGA) he consultado el expediente de la oposición a las cátedras universitarias y en el Archivo General de la Universidad Complutense de Madrid (AGUCM) encontré el expediente académico y el personal de Piedad con el salario que cobraba los años que trabajó allí como Auxiliar de cátedra.

\section{BIBLIOGRAFÍA}

Alcalá Cortijo, P. (1996). Españolas en el CSIC. Presencia y status de las mujeres en la investigación científica española, 1940-1993. En Becerra Conde, G. y Ortiz Gómez, T. (eds.). Mujeres de ciencias. Mujer, feminismo y ciencias naturales, experimentales y tecnológicas. Granada: Universidad de Granada, pp. 61-75.

Alcalá Cortijo, P. y Magallón Portolés, C. (2008). Avances, ruptura y retrocesos: mujeres en las ciencias experimentales en España (19072005). En Romero de Pablos, Santesmases, Cien años de política científica en España. Bilbao: Fundación BBVA, pp. 141-169.

Capel Martínez, M. R. (1986). El trabajo y la educación de la mujer en España (1900-1930). Madrid: Ministerio de Cultura.

Cierva Viudes, P. y Losada, J. (1933). Medidas fotométricas de la reflexión de los Rayos X. Anales de la Sociedad Española de Física y Química, 31, p. 607.

Cierva Viudes, P. y Palacios, J. (1934). Medidas fotométricas de la reflexión de los Rayos X. Anales de la Sociedad Española de Física y Química, 32, p. 391.

Cierva Viudes, P. y Palacios, J. (1935). Factores atómicos absolutos del azufre y del plomo. Anales de la Sociedad Española de Física y Química, 33, pp. 34-38.

Cierva Viudes, P. (1936). Emisión de neutrones por minerales. Anales de la Sociedad Española de Física y Química, 33, pp. 766-769.

Cierva Viudes, P. y Rivoir, L. (1936). Análisis químico por Rayos X. Anales de la Sociedad Española de Física y Química, 34, pp. 770-778. regida en propiedad por una mujer, ya que el puesto de profesoras adjuntas es desempeñado brillantemente por numerosas doctoras" (REP, vol. 11/44, 1953, p. 575). Una muestra de lo largamente que se había mantenido tal prejuicio.

[15] La tesis era "Estudio físico-químico de arcillas y caolines españoles". Un resumen del trabajo fue publicado en una revista de la American Ceramic Society.

[16] La publicación era Cierva Viudes (1955).

[17] El artículo era Cierva Viudes, P., Arroyo, L. (1963), "La superficie del vidrio". Boletín de la Sociedad Española de Cerámica, $\mathrm{n}$ o 2, pp. 95-102. En la primera página se advierte que el artículo era parte de la tesis de Luisa Arroyo.

Cierva, P. de la y Durán, A. (1941). Plateado de espejos. Anales de la Sociedad Española de Física y Química, 37, pp. 177-179.

Cierva, P. de la (1943). Láminas antirreflectoras. Anales de la Sociedad Española de Física y Química, 39, pp. 136-141.

Cierva, P., Durán, A. (1943). Contribución al estudio de la agudeza visual con luz de sodio. Anales de la Sociedad Española de Física y Química, 39, pp. 485-490.

Cierva, P. de la (1944). Láminas antirreflectoras II. Resultados experimentales. Anales de la Sociedad Española de Física y Química, 40, pp. 1031-1033.

Cierva, P. de la y Cacho, F. (1949). Láminas antirreflectoras. Anales de la Sociedad Española de Física y Química, 45, pp. 81-90.

Cierva Viudes, P. (1955). Ensayos de fabricación de vidrio óptico. Madrid: Consejo Superior de Investigaciones Científicas.

Cuesta Bustillo, J., de Prado Herrera, M. L. y Rodríguez Jiménez, F. J. (coords.). (2015) ¿Mujeres sabias? Mujeres universitarias en España y América Latina/Femmes universitaires en Espagne et Amérique Latine. Limoges: Pulim, Presses Universitaires de Limoges.

Eguíbar, M. (2001). Guadalupe Ortiz de Landázuri. Trabajo, amistad y buen humor. Madrid: Palabra.

Flecha García, C. (2010). Profesoras en la Universidad. El tránsito de las pioneras en España. Arenal, 17, 2, pp. 255-297. 
Flecha García, C. (2015). Itinerarios académicos de mujeres en la universidad española. En Cuesta Bustillo, J., de Prado Herrera, M. L. y Rodríguez Jiménez, F. J. (coords.). (2015) ¿Mujeres sabias? Mujeres universitarias en España y América Latina/Femmes universitaires en Espagne et Amérique Latine, pp. 57-81. Limoges: Pulim, Presses Universitaires de Limoges, pp. 57-81.

García Jaramillo, J. (2013) La mitad ignorada (En torno a las mujeres intelectuales de la Segunda República). Madrid: Devenir.

González Ibáñez, C. y Santamaría García, A. (eds.). (2009). Física y Química en la Colina de los Chopos. 75 años de investigación en el edificio Rockefeller del CSIC (1932-2007). Madrid: Consejo Superior de Investigaciones Científicas.

Lerner, G. (2006). A Life of Learning (2005 Charles Homer Haskins Lecture). American Council of Learned Societies. Occasional Papers, 60. Disponible en: http://www.acls.org/Publications/OP/ Haskins/2005_GerdaLerner.pdf

Lewin Simen, R. (1997). Lise Meitner: A Life in Physics. Los Angeles: University of California Press.

Magallón Portolés, C. (1996). ¿Extrañas en el paraíso? Mujeres en las ciencias físico-químicas, en la España de principios del XX. Becerra Conde, G. y Ortiz Gómez, T. (eds.). Mujeres de ciencias. Mujer, feminismo y ciencias naturales, experimentales y tecnológicas. Granada: Universidad de Granada, pp. 33-59.

Magallón Portolés, C. (2001). Científicas en la Sección de rayos X del Rockefeller. En González de Posada, F., González Redondo, F. A. y Trujillo Jacinto del Castillo, D. (eds.). Actas del III simposio "Ciencia y técnica en España de 1898 a 1945: Cabrera, Cajal, Torres Quevedo". Lanzarote: Amigos de la Cultura Científica, pp. 43-52.

Magallón Portolés, C. (2004). Pioneras españolas en las ciencias. Madrid: Consejo Superior de Investigaciones Científicas.

Magallón Portolés, C. (2007). La JAE y las pioneras españolas en las ciencias. En Puig-Samper Mulero, M. A. (ed.). Tiempos de investigación. JAE-CSIC, cien años de ciencia en España. Madrid: Consejo Superior de Investigaciones Científicas, pp. 221-227.

Magallón Portolés, C. (2010a). Del Laboratorio de Investigaciones Físicas a la Meteorología: la primera española doctora en Física, Felisa Martín Bravo. Trayectoria profesional y vital de Felisa Martín Bravo, la primera española doctora en Físicas. En VV.AA. La Junta para Ampliación de Estudios e Investigaciones Científicas en su centenario. Madrid: Publicaciones de la Residencia de Estudiantes e Institución Libre de Enseñanza, pp. 762-791.

Magallón Portolés, C. (2010b). Las mujeres que abrieron los espacios de las ciencias experimentales para las mujeres, en la España del primer tercio del siglo XX. Arenal, 17, 2, pp. 319-347.
Mangini, S. (2001). Las modernas de Madrid. Las grandes intelectuales españolas de la vanguardia. Barcelona: Península.

Martínez Ripoll, M. (2009). La herencia de cien años de cristalografía. En González Ibáñez, C. y Santamaría García, A. (eds.). (2009). Física y Química en la Colina de los Chopos. 75 años de investigación en el edificio Rockefeller del CSIC (1932-2007). Madrid: Consejo Superior de Investigaciones Científicas, pp. 227-234.

Merino Hernández, R. M. (2015). Presencia y ausencia de las mujeres científicas de la Segunda República en $A B C$ y otras fuentes periodísticas. En Cuesta Bustillo, J., de Prado Herrera, M. L. y Rodríguez Jiménez, F. J. (coords.). (2015) ¿Mujeres sabias? Mujeres universitarias en España y América Latina/Femmes universitaires en Espagne et Amérique Latine, pp. 57-81. Limoges: Pulim, Presses Universitaires de Limoges, pp. pp. 225-241.

Montero, M. (2009). La conquista del espacio público. Mujeres españolas en la universidad. Madrid: Minerva.

Mossín Kotin, C. y Losada, J. (1935). Factor atómico del zinc. Anales de la Sociedad Española de Física y Química, 33, 1935, pp. 597-601.

Moya de Gerra, E., Bellanato, J., Flores, A., García-Borge, M. J., Gato, B., Hernández, M. I., Jungclaus, A., Márquez, I., Masegosa, J. y del Olmo, A. (2002). Mujeres en Ciencia y en Tecnologías Físicas en el CSIC. Arbor, 172, 679-680, pp. 549-577. http://dx.doi. org/10.3989/arbor.2002.i679680.1097

Moya de Gerra, E., Bellanato, J., Flores, A., Gato, B., Hernández, M. I., Jiménez, S. Márquez, I., Masegosa, J. y del Olmo, A. (2003) ¿Hay sesgo de género en el área de Físicas del CSIC? Revista Española de Física, 17, 3, pp. 1-7.

Otero Carvajal, L. E. (2012). Las Ciencias Naturales en la Universidad Nacionalcatólica. La reacción antimoderna. Historia del Presente, 20, 2, pp. 51-67.

Palacios, J., Rivoir, L. y Cierva Viudes, P. (1936). Medidas fotométricas de la reflexión de los Rayos X. IV. Comparación de intensidades muy diferentes. Anales de la Sociedad Española de Física y Química, 34, pp. 743-747.

Pérez Fernández-Turégano, C. (2012). José María Otero Navascués. Ciencia y Armada en la España del siglo XX. Madrid: Consejo Superior de Investigaciones Científicas.

Sánchez Ron, J. M. (1994). Miguel Catalán. Su obra y su mundo. Madrid: Consejo Superior de Investigaciones Científicas. 